Physics, Review Article

\title{
Image Quality and Quantitative Accuracy of Small Animal PET Images: Role of Physical Effects Correction and Reconstruction Algorithm
}

\author{
Magdy M. Khalil, PhD \\ Nuclear Medicine Department, King Hamad University Hospital, Kingdom of Bahrain.
}

\begin{abstract}
Positron emission tomography (PET) provides important metabolic information about tracer kinetics. Photon attenuation, scattered radiation, spatial resolution limitation and reconstruction algorithm parameters are variables that influence image quality and quantitative accuracy. The objective of this work was to examine the impact of the combined correction of photon attenuation and scatter on the quality and quantitative accuracy of small animal PET images reconstructed using the analytic filtered back projection algorithm (FBP) and the iterative maximum a posteriori probability (MAP) technique at different values of the smoothing parameter $\beta$.
\end{abstract} Methods. A homogenous water phantomand three different configurations of NEMA image quality phantom were employed using a small animal PET scanner (Inveon $^{\mathrm{TM}}$, Siemens medical solutions, Inc). A $20 \mathrm{~min}$ PET/CT scans were performed for all phantoms using a list mode acquisition. All Data were sorted into 3D sinograms and reconstructed using 2D FBP after Fourier rebinning and also reconstructed using
OSEM3D/MAP ( 2 iteration OSEM3D and 18 iterations using MAP). Figures of merit used were error of tracer estimate, recovery coefficient, percentage standard deviation, spillover ratio and image noise. Results. Based on object geometry and size, FBP with attenuation and scatter correction provides accurate tracer concentration within the range of 5\% and possible improvement can be achieved using MAP reconstruction. Without correction, errors of activity quantitation is significantly high, on average $34.5 \pm 7.2 \%$ Recovery coefficient was also found to improve with proper tuning of the $\beta$ value using MAP reconstruction. Spillover ratio indicated some variations between FBP and MAP in air and water compartments, which might be attributed to the performance of the scatter correction. Noise measurements were superior with MAP reconstruction especially at high $\beta$ values, but this occurs with edge artifacts and slight distortion of some geometric structures. Very small values of the beta have better resolution characteristics but high values have increased smoothing effect and blurring appearance. 
Conclusion: Attenuation correction is important in small animal PET and allows for reliable tracer estimate. Spatial resolution is improved by MAP technique and low $\beta$ values provide high resolution capabilities and recovery coefficients but high values improve noise levels. For a given application, proper selection and employment of the smoothing parameter $\beta$ should be followed. Scatter correction need to be improved especially in large objects or simultaneous acquisition of multiple numbers of animals.

\section{Key words}

Small animal PET, attenuation and scatter correction, maximum a posteriori (MAP), Quantitative accuracy

Corresponding Author: Khalil,M $\quad$ Email: Magdy.khalil@khuh.org.bh

\section{INTRODUCTION}

Small animal positron emission tomography (PET) is an important tool in biomedical and biological imaging research $^{[1,2]}$. There are a number of factors that degrade image quality and reduce quantitative accuracy. Accurate measure of activity concentration in a given region of interest is substantial to derive reliable estimates of tracer kinetics and any subsequent quantitative analysis. Photon attenuation, scatter, random coincidences in addition to limited spatial resolution of PET are among those critical factors in clinical and preclinical setting. Another factor that must be taken into account is the algorithm used in image reconstruction. There might be Little investigations on the impact of these degrading factors on accuracy of quantitative measurements of PET data especially for small animal imaging.

In recent years, there were different preclinical PET scanners released into the market with different performance characteristics and designs ${ }^{[3,4,5,6]}$. Another notable feature is the multimodality approach used by some manufacturers in the configuration of the PET scanner by adding x-ray computed tomography (CT) component to the system. 
Other machines have the capability to add a single photon emission computed tomography (SPECT) module to the imaging gantry so that a one-stop-shop imaging kit can be obtained and installed in a small-sized laboratory.

Inveon $^{\mathrm{TM}}$ (Siemens Medical Solutions, Knoxville, Inc) is a preclinical trimodality imaging scanner that consists of three imaging modules (PET/SPECT/CT) and designed specifically to image small animals such as mice and rats. The system has been evaluated previously and characterized based on National Electrical Manufacturers Association NEMA NU-4 2008 standards, system spatial resolution and sensitivity, normalization, as well as physical performance and scanner throughput. The system was found to provide images of high spatial resolution and detection sensitivity ${ }^{[7,8,9,10]}$. The CT scanner of the system was recently evaluated by our group and was found to have good characteristics that enable to acquire images of high spatial resolution and acceptable noise level [11]. Another feature is the ability to acquire angiographic data using a synchronized respiratory and ECG signals.
Maximum a posteriori (MAP) is among those algorithms used in the inveon reconstruction software. It can reconstruct the PET images with high spatial resolution and better noise properties ${ }^{[12,13]}$. However, the optimal reconstruction setting for small animal PET data is still need further investigations in comparison to filtered back projection(FBP) and under different imaging conditions of attenuation and scatter. Therefore, the objective of this work was to illuminate the combined impact of attenuation and scatter correction together with the reconstruction algorithm on accuracy of tracer concentrations, recovery coefficients, spillover ratio (SOR), and image noise using the NEMA NU-4 image quality phantom designed for small animal PET data.

\section{MATERIALS AND METHODS}

The inveon system was designed to allow researchers acquiring functional and morphological information while keeping the animal in the same position through a unified imaging bed that translate from the CT field of view to the PET module in a user-friendly workflow. System 
specifications are listed in Table 1. The large axial field of view of the PET scanner allows one to scan a whole body mouse in a single session without bed motion or animal translation. It has also the capability to acquire multiple images from the different diagnostic modules and possible combination of SPECT, PET, and CT can be carried out through a workflow software program called inveon acquisition workplace (IAW).
The version of the software used in data acquisition and reconstruction was 1.4. However, image processing and display is implemented using the inveon research workplace (IRW) and both programs were provided by the system manufacturer. The system capability and performance measures were recently evaluated and can be reviewed elsewhere $^{[14]}$

Table 1. The Inveon system (Siemens Medical Solutions) specification

\begin{tabular}{|l|l|}
\hline Detector Crystal material & LSO \\
\hline Crystal size & $1.5 \times 1.5 \times 10 \mathrm{~mm}$ \\
\hline Crystal pitch & $1.59 \mathrm{~mm}$ \\
\hline No. of detector blocks & 64 \\
\hline No. of crystals & 25,600 \\
\hline No. of Detector rings & 80 \\
\hline No. of crystals per ring & 320 \\
\hline Ring diameter & $16.1 \mathrm{~cm}$ \\
\hline Gantry aperture & $12 \mathrm{~cm}$ \\
\hline Axial FOV & $12.7 \mathrm{~cm}$ \\
\hline Transaxial FOV & $10.0 \mathrm{~cm}$ \\
\hline
\end{tabular}

\section{Phantoms}

Four different phantom configurations were used in this study. It was a uniform mouse-sized phantom, NEMA phantom (in air, in the imaging bed, and surrounded by three mouse-sized phantoms). The uniform phantom was a
20 cc syringe filled with homogenous F18 solution and a normal PET/CT workflow was carried out exactly as implemented in our research setting. The total activity in the phantom was 6.5 MBq. 
The NEMA NU-4 phantom was also used in our assessment to the accuracy of activity concentration, image quality, recovery coefficient, noise, and spillover ratio. The phantom consists of a uniform region that contains two identical cylinders as air and water compartments, and 5 rods $(1,2,3,4$ and $5 \mathrm{~mm}$ in diameter) in a solid background.

The phantom was initially filled with a homogenous F18 solution of activity 5.5 $\mathrm{MBq}$ and three sequential studies were carried out. The first acquisition was performed while the phantom was kept without any surrounding medium (i.e in air), the second acquisition was carried out while the phantom placed inside the animal imaging bed which normally used in our research studies. The imaging bed is a multimodal imaging chambers that can be used for CT, PET, SPECT, or MRI data acquisition and manufactured by $\mathrm{m} 2 \mathrm{~m}$ company (Cleveland $\mathrm{OH}$, USA). The third acquisition was done after surrounding the NEMA phantom with three syringes each of the size $20 \mathrm{cc}$; the same size as that used for the uniform phantom experiment.

\section{Acquisition and reconstruction parameters}

The acquisition time was set to $20 \mathrm{~min}$, energy window of 350-650 and timing window of 3.432 nsec and all measurements were undertaken using list mode data acquisition. However, the system is adjustable to other acquisition parameters regarding time/count-based acquisition, energy setting, and coincidence timing window. All data acquisitions were started by CT acquisition using 3 bed positions after laser alignment. The $\mathrm{x}$-ray setting is fixed by the system manufacturer and degree of bed overlap was set to $20 \%$. The voltage and the current of the source were $80 \mathrm{kVp}$ and $500 \mu \mathrm{A}$ respectively using step-and-shoot mode with an x-ray exposure of $200 \mathrm{~ms}$.

After the CT scan, the bed was allowed to move to the PET part of the scanner to start 20 min list mode acquisition the acquired list mode files were then sorted into 3D sinograms for image reconstructions using either 3D iterative techniques, or 2D analytic reconstruction 
using filtered back projection (FBP) after Fourier rebinning ${ }^{[15]}$. Two different reconstruction algorithms were used 1) 2D FBP with and without attenuation and scatter correction, and 2) Maximum a posterior (MAP) with $\beta=0.01,0.1,0.5$ and 1.5 with the constraint of uniform resolution and fast MAP option. The later allows speeding up the reconstruction process with little resolution loss at the edge of the transaxial field of view [16]. The implementation of MAP in IAW software is to start the reconstruction using 2 iterations of OSEM3D followed by 18 iterations of MAP reconstruction. All corrections including normalizations, dead time, random subtraction, attenuation and scatter [17] were applied.

\section{Data analysis}

All decay corrections were taken into account to compute the true value of the phantom activity concentration at the beginning of the PET scan. Estimating the accuracy of tracer concentration was performed by knowledge of the true amounts injected into the phantoms and the measured ones. The difference was obtained and divided by the true value and the result was multiplied by 100 to calculate the percentage error (\% Error) for a given tracer concentration. This calculation has been made to the uniform mouse phantom by drawing 3 cylindrical

Volumes of interest (VOI) such that one VOI at the center of the phantom and the other two were placed at the axial ends of the phantom. The average of the three VOIs was taken to represent the measured tracer concentration in the reconstructed image. The size of the VOIs was $1.1 \mathrm{ml}$. In case of NEMA phantom measurements, a larger VOI of diameter 22.3 and height of $10 \mathrm{~mm}$ was drawn over the uniform phantom region [18]. Standard deviation and average voxel counts were also recorded to calculate percentage standard deviation $(\% \mathrm{SD})$ as a measure of image noise.

For calculation of the recovery coefficients, a cylindrical VOI of height $10 \mathrm{~mm}$ was centered over each rod and the maximum voxel value was compared to the true activity concentration inserted into the phantom to calculate the recovery coefficient for each diameter and for each acquisition. Spillover ratio for air and water compartments were also computed by dividing the mean counts 
obtained from each compartment by the uniform region [18]. The size of the VOI drawn over each compartment was $4 \mathrm{~mm}$ in diameter and $10 \mathrm{~mm}$ in length.

\section{RESULTS}

\section{Accuracy of quantitation}

For the 4 phantom configurations, the simple uniform phantom of homogenous tracer concentration showed the higher accuracy in the reconstructed PET images. In case of absence of attenuation and scatter correction, the error estimated was $24.81 \%$ while the largest error was obtained when the NEMA phantom was surrounded by three objects providing an error of $41.44 \%$. However, when corrections were applied the \% error was significantly improved to $0.02 \%$ and $5.79 \%$ respectively. In the three NEMA phantom experiments performed, the additional structure added in each experiment has resulted in an incremental increase of the error of tracer quantitation. This is consistent with image degradation by the effect of photon attenuation and scatter. However, the application of attenuation and scatter corrections with FBP reconstruction resulted in a significant improvement of tracer activity determination with comparable values among the three data sets, on average $5.96 \%$. .

As can be seen in Table 2, the largest quantitation error was demonstrated by the object of large geometric size (i.e NEMA phantom surrounded by three syringes). All phantom configurations revealed comparable accuracy in tracer activity determination except the uniform syringe phantom, which is not similar to a real imaging scenario. Moving from analytic imaging reconstruction to iterative technique, MAP reconstructed data were better in all NEMA phantoms when compared to FBP combined with correction for the physical effects. Changing the smoothing parameter was also effective in improving the quantitative results of the 4 phantom configurations.

In most imaging scenarios, the uniform simple phantom showed better results than the other NEMA configurations. There was also a trend toward an improved activity quantitation with an increase in the smoothing parameter especially from 0.5 to 1.5 ; however from 0.01 to 0.5 the error values were almost comparable. The uniform phantom 
showed the best results at $\mathrm{MAP}=0.01$ giving an error of measurement of $0.02 \%$ while the NEMA phantom in the imaging bed revealed superior results at $\beta=1.5$ giving an error of $3.5 \%$. Figure 1 shows a line profile drawn over a transverse slice of the uniform phantom for data corrected and uncorrected for photon attenuation and scatter using FBP reconstruction.

Table 2. Accuracy of activity concentration using different objects. Percentage error was calculated by subtracting the measured value from the truth and dividing the results by the true value for the homogenous uniform phantom and the three different NEMA phantoms, in air (NEMA-A), in the animal bed (NEMA-B) and surrounded by 3 syringes (NEMA+3 Syr).

\begin{tabular}{|c|c|c|c|c|}
\hline & Uniform phantom & NEMA-A & NEMA-B & NEMA+3Syr \\
\hline FBP $_{\text {no corr }}$ & 24.81 & 34 & 37.7 & 41.55 \\
\hline FBP $_{\text {at }+\mathrm{sc}}$ & 0.02 & 5.20 & 6.89 & 5.79 \\
\hline MAP 0.01 & 0.21 & 3.5 & 5.5 & 5.1 \\
\hline MAP 0.1 & 0.02 & 3.8 & 5.5 & 4.9 \\
\hline MAP 0.5 & -0.12 & 3.13 & 4.9 & 4.2 \\
\hline MAP 1.5 & -2.08 & 1.8 & 3.5 & 2.7 \\
\hline
\end{tabular}

\section{Spatial Resolution}

Recovery coefficient measurements for the 3 NEMA phantom experiments showed similar patterns but the NEMA phantom surrounded by three syringes showed the worst results especially when data are not corrected for physical effects. In all conditions, the rod of
Largest diameter (i.e. $5 \mathrm{~mm}$ ) showed a recovery coefficient of less than 100\% using $\mathrm{FBP}_{\mathrm{at}+\mathrm{sc}}$. On averages, the $\mathrm{RC}$ was $0.90 \pm 0.02$ for data corrected, while it was $0.60 \pm 0.03$ for data not corrected as shown in Figure 2. 


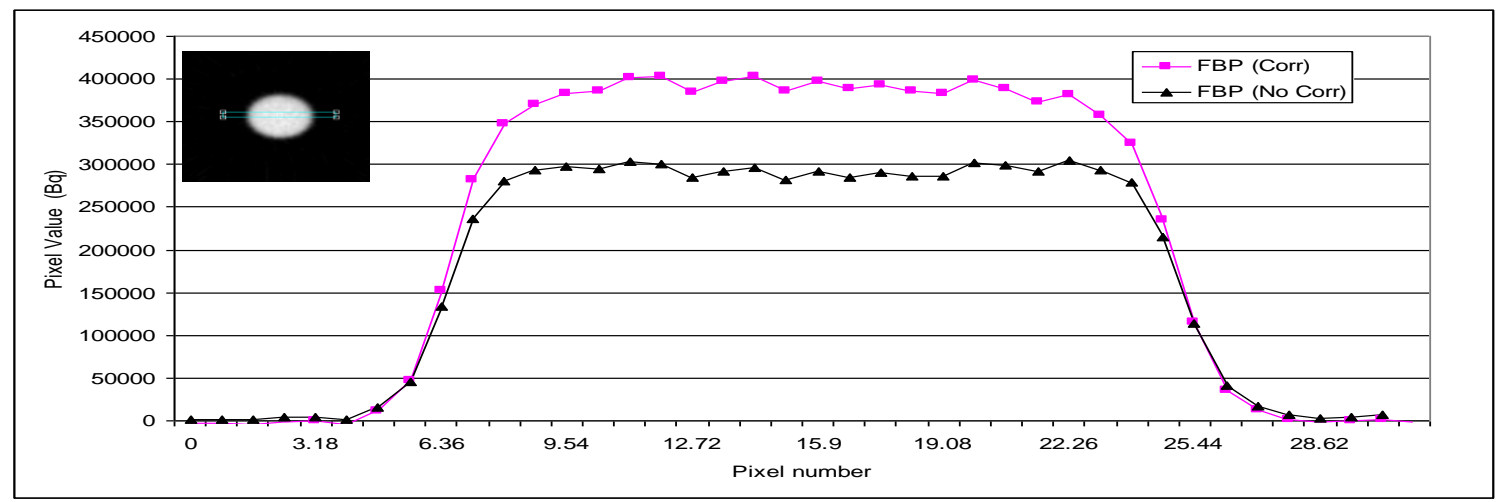

Figure 1. A line profile drawn over a transverse slice of the simple homogenous phantom for data corrected and uncorrected for photon attenuation (data reconstructed using FBP)
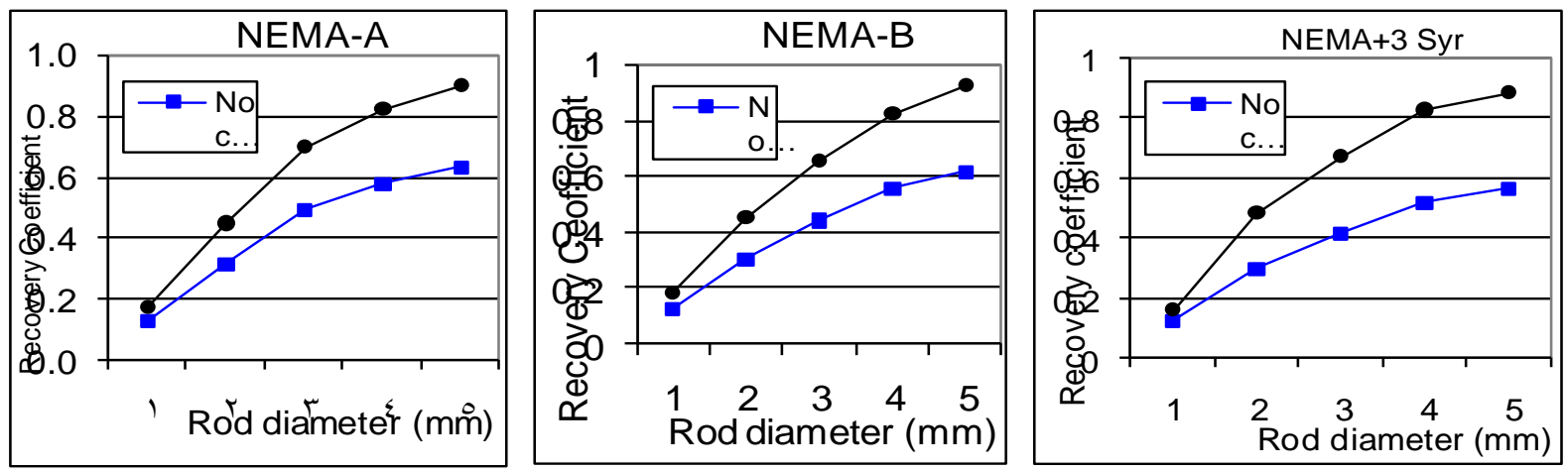

Figure 2. Recovery coefficients measured using the NEMA phantom in air (NEMA-A), in the animal bed (NEMA-B) and surrounded by 3 syringes (NEMA+3 Syr).

In MAP reconstruction, adjusting the $\beta$ parameter was effective in improving the spatial resolution demonstrated by enhancement of the RCs, see Figure 3. For rod size of $4 \mathrm{~mm}$ in diameter the true signal was almost recovered in all NEMA phantom studies using $\beta$ value of 0.01 . On average, the $\mathrm{RC}$ was $0.98 \pm 0.03$ for that rod size. However, other $\beta$ values were inferior to achieve such a recovery at the same rod size. At rod size of $5 \mathrm{~mm}$, a $\beta$ value of 0.1 provided a very high PET signal recovery with an average of $1.01 \pm 0.01$ across the three NEMA phantoms. Figure 4 shows a transverse image at the level of the rods of the NEMA phantom using different values of the hyperparameter $\beta$ together with data corrected and uncorrected using FBP reconstruction. 

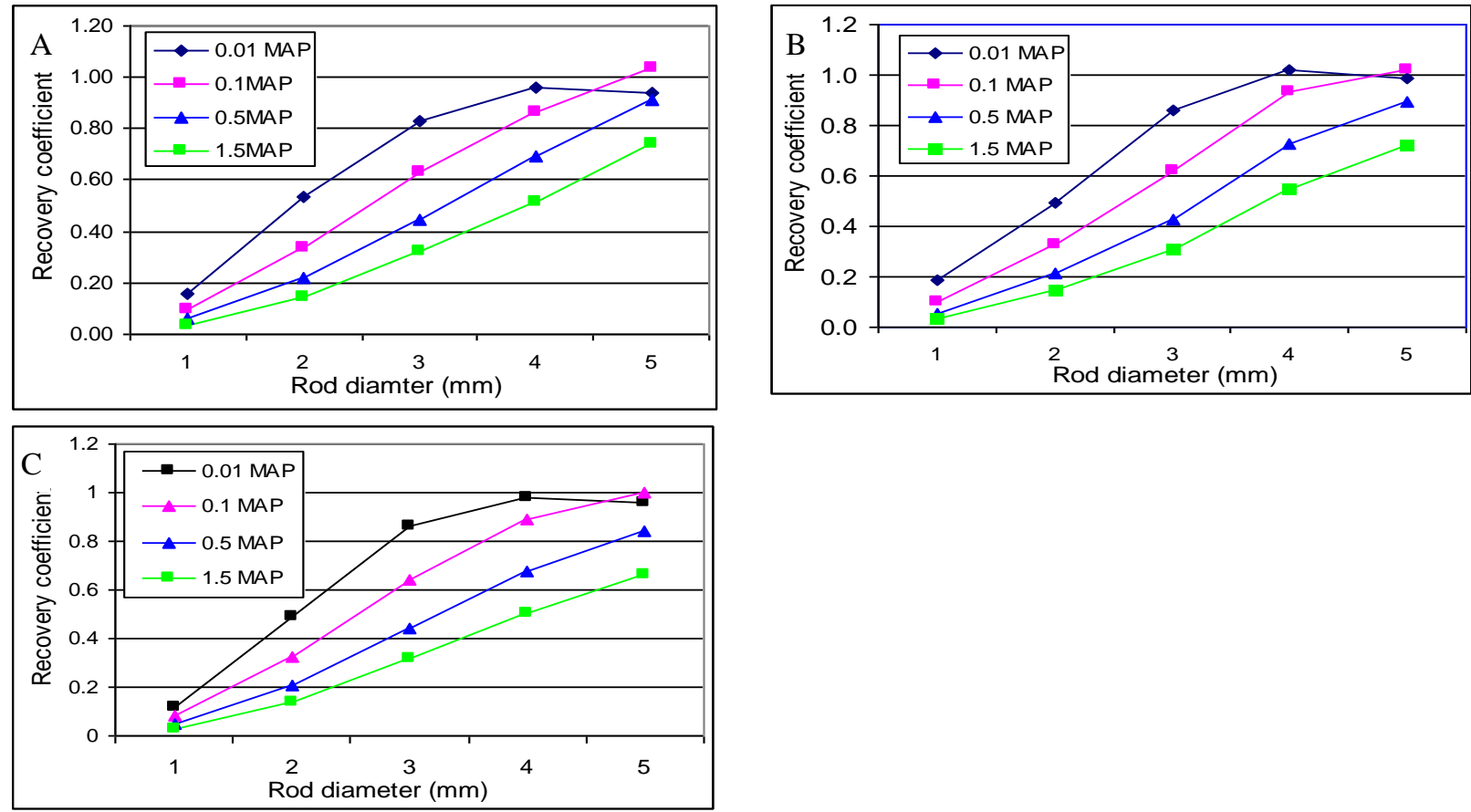

Figure 3. Recovery coefficients measured using the NEMA phantom in air (A), in the animal bed (B) and surrounded by 3 syringes (c) using MAP reconstruction at $\beta=0.01,0.1,0.5$ and 1.5

$\mathrm{FBP}_{\mathrm{No}} \mathrm{Corr}$
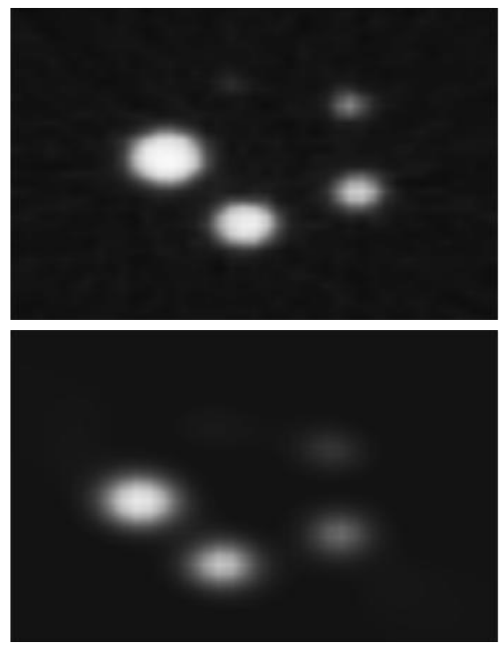

MAP, $\beta=0.5$
$\mathrm{FBP}_{\mathrm{At}+\mathrm{SC}}$
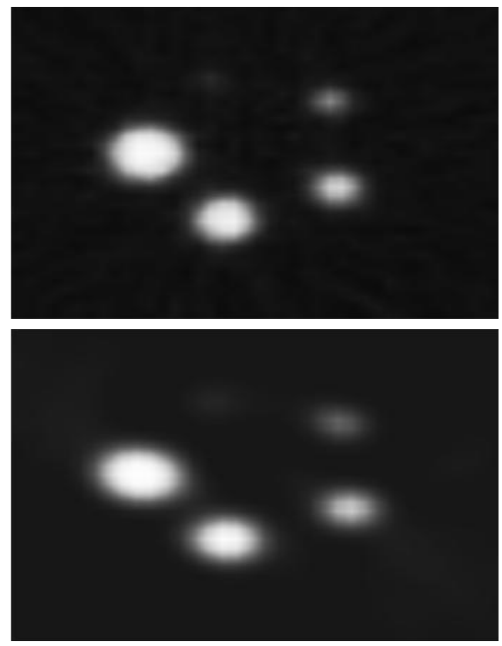

MAP, $\beta=0.1$
MAP, $\beta=1.5$
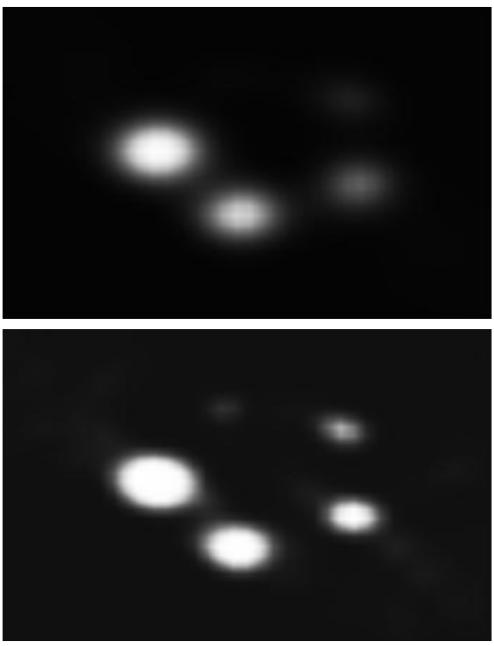

MAP, $\beta=0.01$

Figure 4. The impact of the reconstruction algorithm on the quality of PET images acquired for NEMA phantom placed in the animal bed. Note the slight geometric distortion of the largest rods (i.e 4 and $5 \mathrm{~mm}$ for $\beta$ at 0.01 and 0.1 ). 
Spillover measurements are shown in figure 5. In all phantoms, spillover ratios were the highest in data not corrected for attenuation and scatter with FBP reconstruction. This measure actually evaluates the impact of scatter correction on image quality and should serve to reduce the SOR to the minimum. Data corrected for attenuation and scatter and reconstructed by FBP were in some instances better than iterative MAP reconstruction. However, this was consistent in air compartments than water filled compartments.

\section{Image Noise}

Calculating the coefficient of variation or what is called $\% \mathrm{SD}$ as a measure of image noise is shown in figure 6. One can notice that as the imaged object increases in size, there is a corresponding increase in the noise component in data not corrected for attenuation and scatter. However, in all phantoms these values were improved after correction and reconstruction using FBP. MAP reconstruction showed an improvement in image noise as the $\beta$ value was increased being the highest at $\beta=0.5$ and 1.5 , bearing in mind that uniform resolution option was selected in this study.

\section{DISCUSSION}

In vivo small animal PET imaging has gained a great interest and large number of applications in biomedical and biological preclinical research. One of the features that characterize the reconstructed PET data is the ability to quantify tracer concentration in a particular area of interest. The objective of the present study was to look at different factors such as the impact of combined effects of attenuation and scatter correction on the calculation of the tracer quantitative accuracy, recovery coefficients, image noise, and searching for differences that exist between FBP and MAP reconstruction using the standard NEMA NU-4 mouse imagequality phantom.

In terms of activity quantitation in a homogenous medium, it was shown that FBP can provide very accurate results. MAP reconstruction can also provide similar results while being able to overestimate the true values when the smoothing parameter was getting higher. However, for the three NEMA 

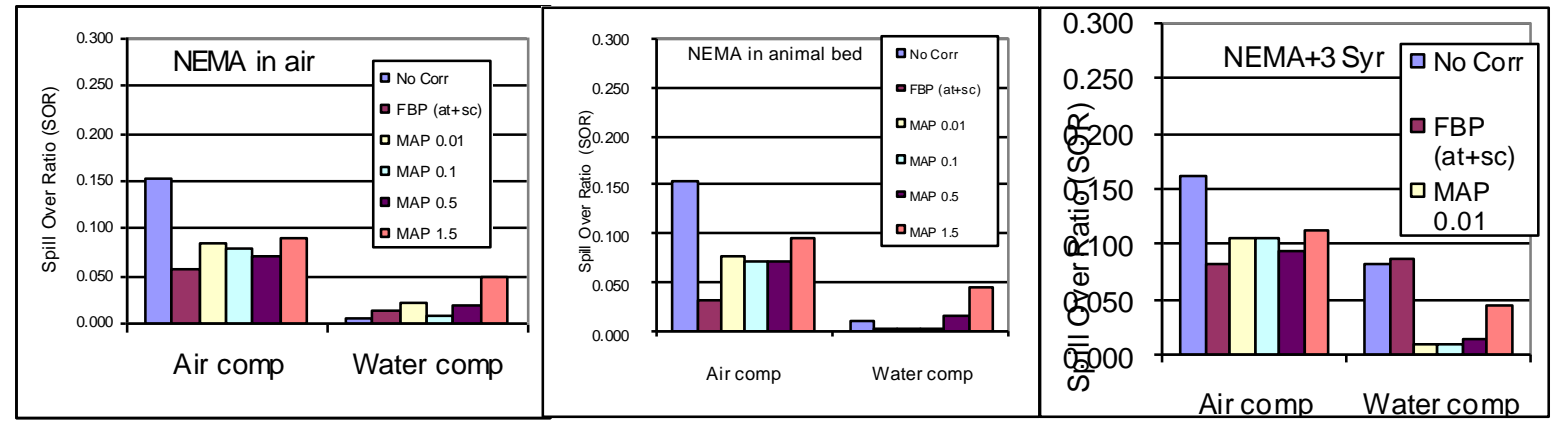

Figure 5. Spillover ratio in air and water compartments under different conditions of attenuation and scatter using FBP and MAP reconstruction algorithms.

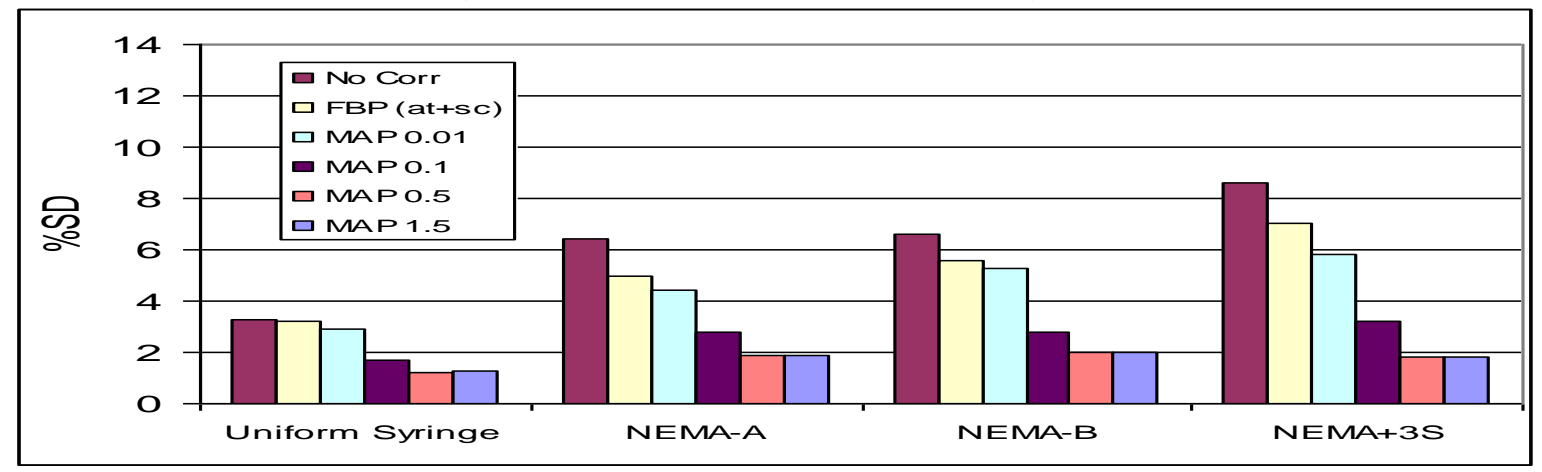

Figure 6. Percent standard deviation as a function of attenuation medium, object size, and reconsrtruction algorithm.

A
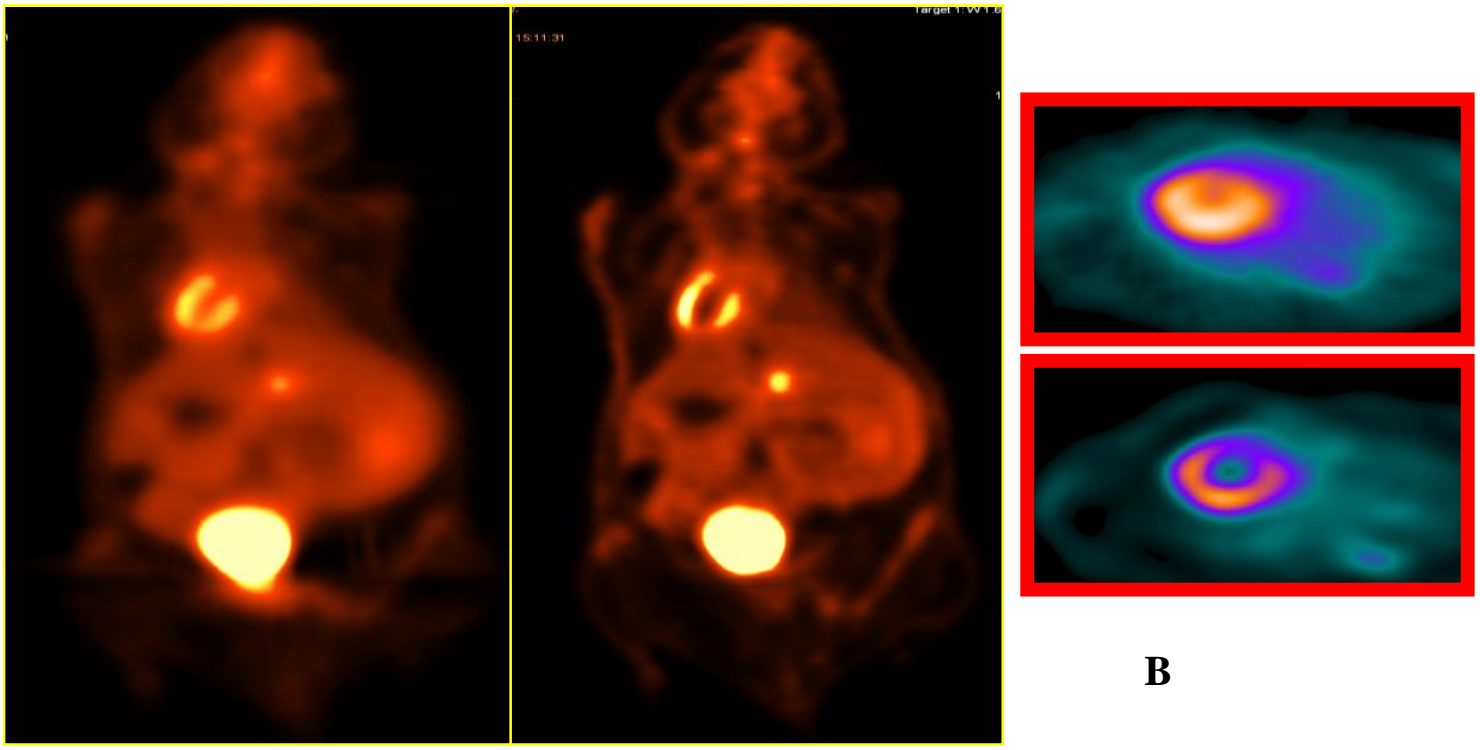

B

Figure 7.,(A) two coronal images have been reconstructed using 2D FBP and 0.01 MAP algorithms showing good spatial resolution and contrast enhancement.(B) The two myocardial short axis images also demonstrate how MAP reconstruction can significantly improve myocardial contrast and image quality. 
configurations, $\mathrm{FBP}_{\mathrm{at}+\mathrm{sc}}$ reconstructions revealed an average error of $5.96 \pm 0.85 \%$. These measurements were also improved when MAP reconstruction was used particularly at large $\beta$ ( 0.5 and 1.5$)$

The NEMA phantom is different from a homogenous cylindrical phantom and thus the error values were mildly higher than a simple activity distribution. The errors for data not corrected for photon attenuation and scatter were extremely high, on average, $34.5 \pm 7.1 \%$ revealing that attenuation correction has a substantial effect on tracer activity quantitation even in mouse PET imaging. These values are consistent with some previous reports showing that photon attenuation could reduce tracer estimate by $40 \%{ }^{[19]}, 26.4 \%$ in mouse-sized phantom and $38.5 \%$ in rat-sized phantom ${ }^{[20]}$. CT-based attenuation correction has several advantages over other methods which rely on measured or calculated attenuation coefficients. Among those is the significant reduction of noise propagation from transmission data to the emission images, fast scanning time, together with anatomical correlation with the functional images. Drawbacks of CTbased attenuation correction can be registration errors between the transmission scan and the emission data, which can result in non-trivial error $>10 \%$ for $1.2 \mathrm{~mm}$ misalignment [21]. However, the PET and CT data in this study was matched automatically based on a predefined transformation matrix acquired using PET and CT scans as recommended by the system manufacturer.

Measuring the response of the $\mu$ PET scanner versus objects of diameter size in the range of the full width at half maximum (FWHM), or spatial resolution of the imaging system, provides an insight of the recovery coefficient profile and can be used to correct for partial volume effect ${ }^{[22]}$. It was shown that correction of partial volume effects using recovery coefficient measurements allowed for improvement of semiquantitative data in a wide range of subcutaneous and visceral tumors except in large necrotic lesions although no attenuation correction was performed ${ }^{[23]}$. As noted from the results, there was an increasing trend toward restoring the whole PET signal as the object size increased in diameter. Also it was noted that the impact of corrections is higher in 
large than the small phantom rods Further, iterative reconstruction was able to significantly improve the recovery coefficients than FBP reconstruction. MAP reconstruction at $\beta$ of 0.01 or 0.1 provided better recovery coefficients than higher $\beta$ values.

This smoothing parameter (or hyperparameter) was shown to control image quality and can be tuned for a given application to improve the detection or estimation task. However, it was reported that at very low values of $\beta$, an activity depression around edges can occur resulting in image distortion and quantitative errors ${ }^{[10]}$. Another recent report reveled that small $\beta$ values have better point spread function (PSF) measurements but with less accuracy as the Chi square tends to increase. The authors therefore have used $\beta=0.05$ to ensure that the PSF have a Gaussian shape to incorporate it in their proposed partial volume correction scheme ${ }^{[24]}$. Another observation revealed by this study is that at very small values of $\beta$ the normal spherical shape of the rods was slightly distorted as shown in figure 4 where one can notice that the rod image has become slightly elliptical in the diagonal direction especially at $\beta=0.1$ and 0.01 .

Measuring the spillover for water and air compartments revealed different results with FBP and MAP reconstruction. In air compartment the SOR was significantly higher than those measured for water compartment. Increasing the object size was found to increase the count spillover from region to another due to the fact that more scattering centers do exist in the emission path. $\mathrm{FBP}_{\mathrm{at}+\mathrm{sc}}$ was helpful to reduce the SOR in all air compartments when compared to MAP reconstruction. However, in water compartment, there were some differences as some $\beta$ values were able to outperform $\mathrm{FBP}_{\mathrm{at}+\mathrm{sc}}$ in some conditions. This may reflect and highlight the combined effect of the performance of scatter correction and selection of the smoothing parameter $\beta$. Further, this is to some extent in agreement to previous reports where scatter correction with MAP was not recommended while it was useful with other reconstruction algorithms like FBP and OSEM ${ }^{[10]}$. Reduction of SOR has practical importance to improve lesion contrast and also in quantitative tracer kinetic analysis. Correction of count 
spillover from region to another is critically important in image derived input function as it confounds the kinetic model to reliably derive the rate constants of a dynamic physiological process ${ }^{[25]}$. Image noise was substantially improved by use of iterative reconstruction especially at higher $\beta$ values; however, this occurs with smoothing effect on fine details and some resolution loss. Hence, the proper $\beta$ value for a particular imaging task should be optimally defined in terms of a contrastnoise trade-off regimen. MAP reconstruction provides several good features for the quality of the micro-PET images and has been evaluated previously in comparison to analytic as well as iterative techniques. It was shown that MAP algorithms is able to recover the true activity ratio better than FBP in addition to its superior resolution properties together with better noise measurements and mitigation of partial volume effects

\section{CONCLUSION}

The present study showed the importance of the combined effect of attenuation and scatter correction in improving the overall tracer estimate in small animal $\mu$ PET images. Recovery coefficients calculation is also affected by such corrections and
$[10,12]$. Figure 7 displays two images reconstructed using FBP and MAP at $\beta$ $=0.01$ for the same animal . who received $10 \mathrm{MBq}$ FDG intravenously and acquisition commenced immediately for a time course of $1 \mathrm{hr}$. Looking at the results one can notice that the imaging bed has no major effect in reducing the accuracy of measurements or degrading image visual quality. However, using the scanner for simultaneous acquisition of multiple number of mouse experiments need to addressed for a given application ${ }^{[26]}$ .Further work is warranted to improve the performance of the scatter correction and evaluate its performance separately (with and without attenuation correction) in small animal PET imaging and in combination with different reconstruction algorithms. This is currently being investigated in our lab using objects of different geometry including rat phantom models and xenograft implanted tumors.

significant improvement can be seen when using MAP reconstruction. The shape of the recovery coefficient curve can also be altered based on particular iterative reconstruction setting and hence this needs to be taken into account when correction 
of partial volume is sought. Image noise and resolution properties of the PET images reconstructed with MAP should be defined and optimized for a given imaging task. However, MAP iterative reconstruction provides images with high quantitative accuracy, better resolution

\section{REFERENCES}

1. Cherry SR. In vivo molecular and genomic imaging: new challenges for imaging physics. Phys Med Biol. Feb 7; 49 (3):R13-48; 2004.

2. Phelps ME. PET: the merging of biology and imaging into molecular imaging J Nucl Med. Apr; 41 (4):661-81; 2000.

3. Seidel J, Vaquero JJ, Lee IJ, Green MV. Experimental estimates of the absolute sensitivity of a small animal PET scanner with depth-of-interaction capability," Nuclear Science Symposium Conference Record, 2000 IEEE, vol.3, no., pp.21/57-21/59 vol.3; 2000.

4. Tai YC, Chatziioannou A, Siegel S, Young J, Newport D, Goble RN, Nutt RE, Cherry SR. Performance evaluation of the microPET P4: a PET system characteristics than FBP and image noise. Further work is warranted on the performance of the scatter correction on large subjects with variable and different conditions including source location and surrounding media.

dedicated to animal imaging. Phys Med Biol; 46:1845-1862; 2001.

5. Christof Knoess, Norbert Richerzhagen, Rudolf Graf, Klaus Wienhard. Performance evaluation of the microPET R4 PET scanner for rodents. Eur J Nucl Med; 2003.

6. Prasad R, Ratib O, Zaidi H. Performance evaluation of the FLEX triumph X-PET scanner using the national electrical manufacturers association NU-4 standards J Nucl Med. 2010 Oct;51(10):1608-15. Epub Sep 16; 2010.

7. Constantinescu CC, Mukherjee J. Performance evaluation of an Inveon PET preclinical scanner. Phys Med Biol. May 7; 54 (9):2885-99. Epub 2009 Apr $21 ; 2009$. 
8. Bao Q, Newport D, Chen M, Stout DB, Chatziioannou AF. Performance evaluation of the inveon dedicated PET preclinical tomograph based on the NEMA NU-4 Standards. J Nucl Med. 2009 Mar; 50 (3): 401-8. Epub Feb 17; 2009.

9. Visser EP, Disselhorst JA, Brom M, Laverman P, Gotthardt M, Oyen WJ, Boerman OC.Spatial resolution and sensitivity of the Inveon small-animal PET scanner. J Nucl Med. Jan; 50 (1): 139-47; 2009.

10. Visser EP, Disselhorst JA, van Lier MG, Laverman P, de Jong GM, Oyen WJ, Boerman OC. Characterization and optimization of image quality as a function of reconstruction algorithms and parameter settings in a Siemens Inveon small-animal PET scanner using the NEMA NU 4-2008 standards. Nucl. Instr. and Meth. A Volume 629, Issue 1, 11 February, Pages 357-36; 2011

11.Khalil MM, Gsell W, Myers M. Performance characteristics of the Inveon micro-CT scanner in small animal imaging. [Abstract] 0327B:3a. WMIC proceeding, Kyoto, Japan, 8-11 Sep; 2010.

12. Chatziioannou A, Qi J, Moore A, et al. Comparison of 3-D maximum a posteriori and filtered backprojection algorithms for high-resolution animal imaging with microPET. IEEE Trans Med Imaging. 19:507-512; 2000.

13. Qi J, Leahy RM, Cherry SR, Chatziioannou A, Farquhar TH. Highresolution 3D Bayesian image reconstruction using the microPET small-animal scanner. Phys Med Biol.; 43:1001-1013; 1998.

14. Magota K, Kubo N, Kuge Y, Nishijima KI, Zhao S, Tamaki N. Performance characterization of the Inveon preclinical small-animal PET/SPECT/CT system for multimodality imaging. Eur J Nucl Med Mol Imaging. Dec 10. [Epub ahead of print]; 2010.

15. Defrise M, Kinahan PE, Townsend DW, Michel C, Sibomana M, Newport DF. Exact and approximate rebinning 
algorithms for 3-D PET data. IEEE Trans

Med Imag. 16:145-158; 1997.

16. Cho S; Li Q; Ahn S; Bai B; Leahy RM. Iterative Image Reconstruction Using Inverse Fourier Rebinning for Fully 3-D PET IEEE Trans. Medical Imaging, 26 (5):745-756; 2007.

17. Watson CC. New, faster, imagebased scatter correction for 3D PET. IEEE Trans Nucl Sci.; 47:1587-1594; 2000.

18. National Electrical Manufacturers Association (NEMA). Performance Measurements for Small Animal Positron Emission Tomographs (PETs). NEMA Standards Publication NU 4-2008. Rosslyn, VA: NEMA; 2008.

19. Visser EP, Van Dalen JA, Laverman P, Newport D, Corstens FHM, Oyen WJG, et al. Evaluation of attenuation correction in the Inveon preclinical PET scanner using two opposite, rotating 57Co sources. Eur J Nucl Med Mol Imaging; 34:S227; 2007.
20. Chow PL, Rannou FR, Chatziioannou AF. Attenuation correction for small animal PET tomographs. Phys Med Biol. Apr 21; 50 (8): 18; 2005.

21. Chow PL, Stout DB, Komisopoulou E, Chatziioannou AF. A method of image registration for small animal, multi-modality imaging. Phys Med Biol. 2006 Jan 21;51(2):379-90. Epub Jan 4; 2006.

22. Hoffman EJ, Huang SC, Phelps ME. Quantitation in positron emission computed tomography: 1. Effect of object size. J Comput Assist Tomogr; 3:299-308; 1979.

23. Aide N, Louis MH, Dutoit S, Labiche A, Lemoisson E, Briand M, Nataf V, Poulain L, Gauduchon P, Talbot JN, Montravers F. Improvement of semiquantitative small-animal PET data with recovery coefficients: a phantom and rat study. Nucl Med Commun. Oct; 28 (10): 813-22; 2007. 
24. Dumouchel T, de Kemp RA. 18F-FDG PET studies. J Nucl Med. 2008 Analytical-Based Partial Volume Apr; 49(4):606-14. Epub Mar 14; 2008. Recovery in Mouse Heart Imaging,". IEEE Transactions on Nuclear Science, vol. 58, no.1, pp.110-120, Feb; 2011.

26. Aide N, Desmonts C, Briand M, Meryet-Figuiere M, Poulain L. Highthroughput small animal PET imaging in 25. Fang YH, Muzic RF Jr. Spillover and cancer research: evaluation of the partial-volume correction for imagecapability of the Inveon scanner to image derived input functions for small-animal four mice simultaneously. Nucl Med Commun. Oct; 31(10):851-8; 2010. 
